\title{
hsa_circ_0008234 inhibits the progression of lung adenocarcinoma by sponging miR-574-5p
}

\author{
Wei Jiang ${ }^{1}$, Yaozhou He${ }^{2}$, Zijian Ma ${ }^{2}$, Yu Zhang ${ }^{1}$, Chengpeng Zhang ${ }^{1}$, Nianpeng Zheng ${ }^{1}$ and Xing Tang ${ }^{1}$
}

\begin{abstract}
circRNAs are a novel type of noncoding RNA (ncRNA) that have been identified as an important regulator of gene expression and play a part in the progression of various diseases. However, the function of circ_0008234 in lung adenocarcinoma (LUAC) remains unknown. Through the GEO (Gene Expression Omnibus) database, circ_0008234 was first found to be downregulated in LUAC tissues. It could inhibit cell growth and accelerate apoptosis in vitro and in vivo. In terms of its possible mechanism, circ_0008234 mainly was present in the cytoplasm and competed with miR-574-5p to regulate RND3 (Rho family GTPase 3). Our results revealed that circ_0008234 inhibited the progression of LUAC through a competing endogenous RNA (ceRNA)-based mechanism and provided potential biomarkers and therapeutic targets for LUAC treatment.
\end{abstract}

\section{Introduction}

Lung cancer (LC) remains one of the most common malignant tumors in the world, and non-small cell lung cancer (NSCLC) is the predominant type (approximately $85 \%$ ) of LC, including lung adenocarcinoma (LUAC) ${ }^{1}$. LUAC accounts for approximately $50 \%$ of all types of LC, and its number has been increasing year by year, especially among women and young people ${ }^{2}$. At present, anatomical surgical resection is the most safe and effective method for patients with early-stage LUAC, including lobectomy and sublobectomy ${ }^{3}$. Due to the various clinical manifestations of advanced-stage LUAC, systemic and local therapies (chemotherapy, immunotherapy, radiation therapy, etc.) are considered the best option for symptom control basing on the principle of multimodal therapy ${ }^{4,5}$. Although LUAC's treatment model has been greatly developed, the 5-year overall survival of patients with LUAC remains unsatisfactory $(<20 \%)^{6}$. Therefore, in order to improve the early diagnosis and treatment of

\footnotetext{
Correspondence: Xing Tang (tangx81@163.com)

'Department of Thoracic Surgery, The First Affiliated Hospital of Soochow

University, Suzhou, Jiangsu Province, P. R. China

${ }^{2}$ Department of Thoracic Surgery, The First Affiliated Hospital of Nanjing

Medical University, Nanjing, Jiangsu Province, P. R. China

These authors contributed equally: Wei Jiang, Yaozhou He

Edited by M. V. Niklison Chirou
}

LUAC, it is urgent to find new biomarkers and therapeutic targets.

Unlike traditional linear RNA, circular RNA (circRNA) has a closed loop structure, which is not affected by RNA exonuclease and is not easily degraded ${ }^{7}$. Increasing evidence shows that circRNAs are frequently deregulated in various cancers and act as tumor suppressors or oncogenes ${ }^{8}$. To date, an increasing number of circRNAs have been found to function as competing endogenous RNAs (ceRNAs) in various cancers, including LUAC ${ }^{9}$. The ceRNA hypothesis was formally proposed in 2011 and describes a large-scale regulatory mechanism that regulates gene expression through the transcriptome ${ }^{10}$. MicroRNAs (miRNAs) not only affect the transcription and stability of RNA at the posttranscriptional level by binding to target genes but also affect miRNAs, which can be called "circRNA $\rightarrow$ miRNA $\rightarrow$ RNA" interactions ${ }^{11}$. Studies have shown that circRNA_104348 can promote hepatocellular carcinoma progression by modulating the miR-187-3p/RTKN2 (Rhotekin 2) axis ${ }^{12}$. Circ_0000020 can elevate the expression of PIK3CA (phosphatidylinositol 3-kinase C) and facilitate the malignant phenotypes of glioma cells by targeting miR-142-5 $\mathrm{p}^{13}$. An increasing number of circRNAs have been found to be unbalanced in LUAC and can be used as therapeutic targets in the

\section{(c) The Author(s) 2021}

(c) (i) Open Access This article is licensed under a Creative Commons Attribution 4.0 International License, which permits use, sharing, adaptation, distribution and reproduction cc) in any medium or format, as long as you give appropriate credit to the original author(s) and the source, provide a link to the Creative Commons license, and indicate if changes were made. The images or other third party material in this article are included in the article's Creative Commons license, unless indicated otherwise in a credit line to the material. If material is not included in the article's Creative Commons license and your intended use is not permitted by statutory regulation or exceeds the permitted use, you will need to obtain permission directly from the copyright holder. To view a copy of this license, visit http://creativecommons.org/licenses/by/4.0/. 


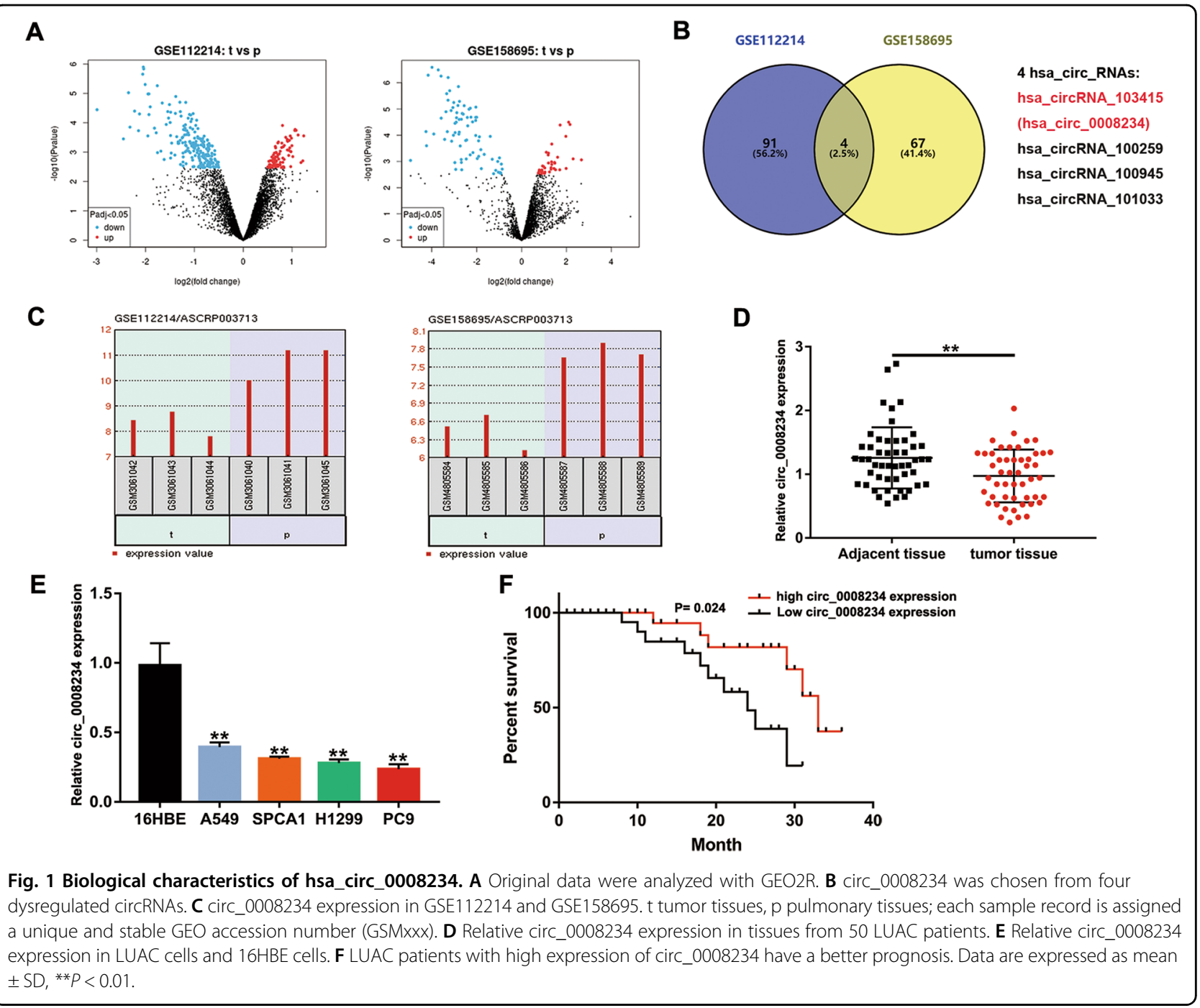

future. In LUAC cells, for example, hsa_circ_0027446 could promote metastasis and epithelial-mesenchymal transition (EMT), while low expression of circ_0018414 could influence proliferation, stemness, and apoptosis ${ }^{14,15}$. hsa_circ_0008234 is transcribed from chr3:7109047871102924, where the FOXP1 (forkhead box P1) gene is located, approximately $587 \mathrm{bp}$ in length. In gallbladder cancer, circ_0008234 contributes to tumor progression and the Warburg effect by regulating PKLR (pyruvate kinase $L / R)^{16}$. However, there are few studies on the regulatory mechanism of circ_0008234 in LUAC.

Our research first showed that circ_0008234 was markedly decreased in LUAC tissues and cell lines. Furthermore, circ_0008234 overexpression (OE) suppressed proliferation and facilitated apoptosis in vitro and in vivo. Furthermore, circ_0008234 could sponge miR-574-5p to regulate RND3 (Rho family GTPase 3 ) to affect the progression of LUAC.

\section{Results}

The circ_0008234 expression level was decreased in LUAC tissues and cells

Raw microarray data of NSCLC patients were downloaded from Gene Expression Omnibus (GEO), including GSE112214 $(n=6)$ and GSE158695 $(n=6)$. To obtain differentially expressed circRNAs, data were analyzed with GEO2R (Fig. 1A). Since there was no intersection of abnormally upregulated circRNAs, we chose circ_0008234 as the research object from the intersection of abnormally downregulated circRNAs (Fig. 1B). Subsequently, we extracted the original data of circ_0008234 from GSE112214 and GSE158695 and further verified that circ_0008234 expression in tumor tissues of NSCLC patients was observably lower than that in normal tissues (Fig. 1C). To further investigate whether this phenomenon exists in LUAC, 50 patients who met the enrollment criteria were randomly selected, and it was found that the 
expression of circ_0008234 in the tumor tissues was lower than that in adjacent tissues (Fig. 1D). Furthermore, circ_0008234 was significantly abnormally downregulated in the LUAC cell lines (H1299, SPCA1, A549, and PC9)

Table. 1 Expression of circ_0008234 according to patients' clinical features.

\begin{tabular}{|c|c|c|c|}
\hline \multirow[t]{2}{*}{ Factors } & \multicolumn{2}{|c|}{$\begin{array}{l}\text { circ_0008234 } \\
\text { expression }\end{array}$} & \multirow[t]{2}{*}{$P$ value } \\
\hline & High & Low & \\
\hline \multicolumn{4}{|l|}{ Gender } \\
\hline Male & 12 & 13 & 0.568 \\
\hline Female & 10 & 15 & \\
\hline \multicolumn{4}{|l|}{ Age (years) } \\
\hline$\geq 50$ & 8 & 17 & 0.087 \\
\hline$<50$ & 14 & 11 & \\
\hline \multicolumn{4}{|c|}{ Distant metastasis } \\
\hline Yes & 5 & 7 & 0.851 \\
\hline No & 17 & 21 & \\
\hline \multicolumn{4}{|c|}{ Tumor size $(\mathrm{cm})$} \\
\hline$>3$ & 7 & 18 & $0.023^{*}$ \\
\hline$\leq 3$ & 15 & 10 & \\
\hline \multicolumn{4}{|c|}{ Lymph node metastasis } \\
\hline Yes & 8 & 8 & 0.558 \\
\hline No & 14 & 20 & \\
\hline
\end{tabular}

$* P<0.05$ compared with the 16HBE cell line (Fig. 1E). Finally, the correlations between circ_0008234 expression levels and clinical and pathological features were considered. It was found that circ_0008234 expression was remarkably correlated with the tumor size of LUAC patients (Table 1). The prognosis of LUAC patients with high expression of circ_0008234 was better than that of patients with low expression (Fig. 1F). In conclusion, circ_0008234 was downregulated in LUAC, and low expression of circ_0008234 predicted poor prognosis in LUAC patients.

\section{The biological characteristics of circ_0008234}

Based on the circ_0008234 expression of LUAC cell lines, H1299 and PC9 cells were selected for further study. In the RNase R treatment assay, the mRNA expression of FOXP1 was significantly decreased, while the circ_0008234 expression was not significantly changed (Fig. 2A). This study showed that circ_0008234 is robust and has stable expression in the cells. Then determining the subcellular location of circ_0008234 became a top priority. Next, we studied the localization of circ_0008234 in LUAC cells. Figure 2B, C showed that circ_0008234 was present in the cytoplasm and nucleus of H1299 and PC9 cells. The implication of these results was that circ_0008234 could stably exist in the cytoplasm and nucleus of LUAC cells, providing a basis for its biological functions.

\section{circ_0008234 OE influenced cell proliferation and apoptosis}

To discover the role of circ_0008234, circ_0008234 OE plasmids and negative control (NC) plasmids were used to

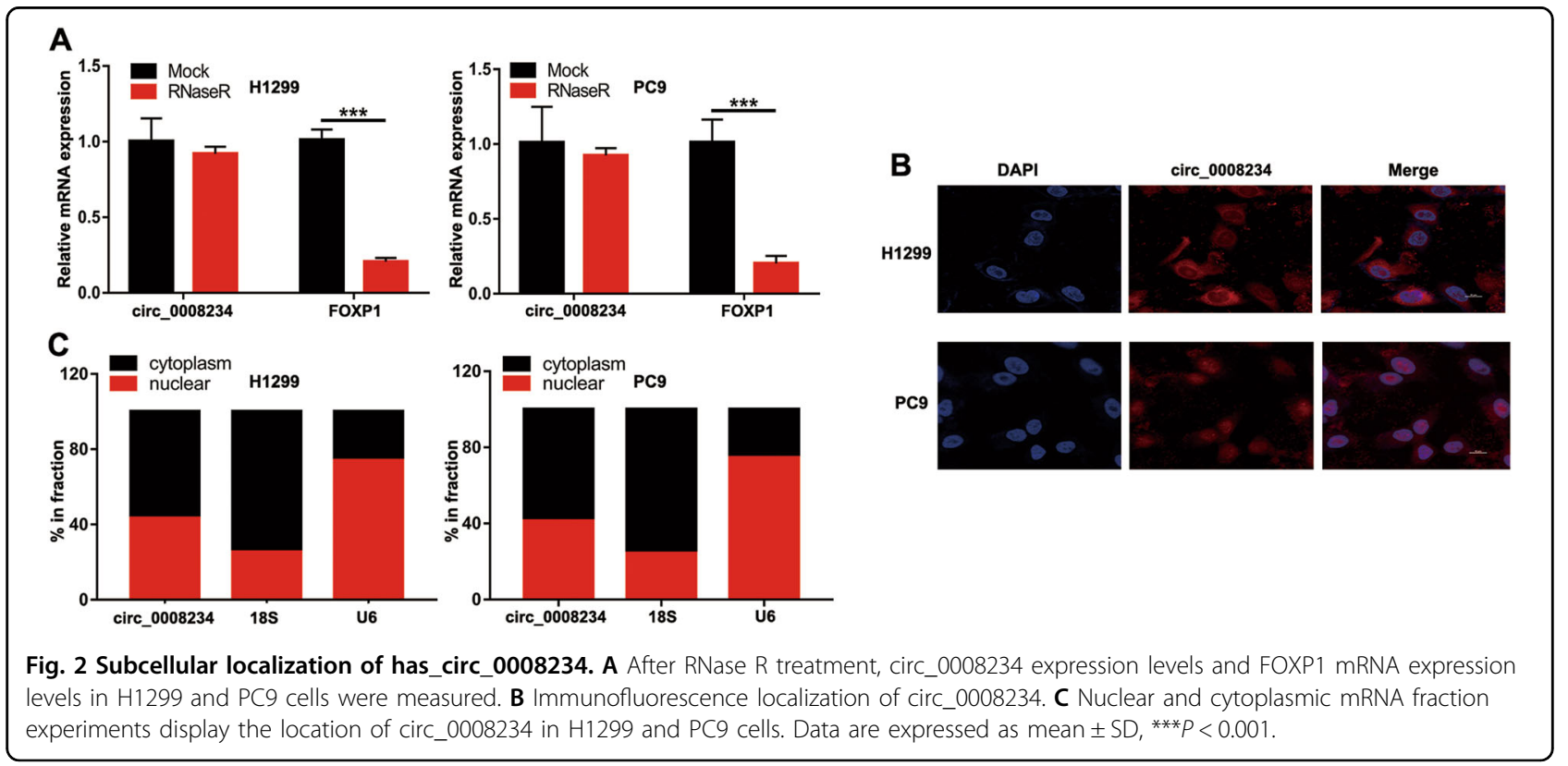




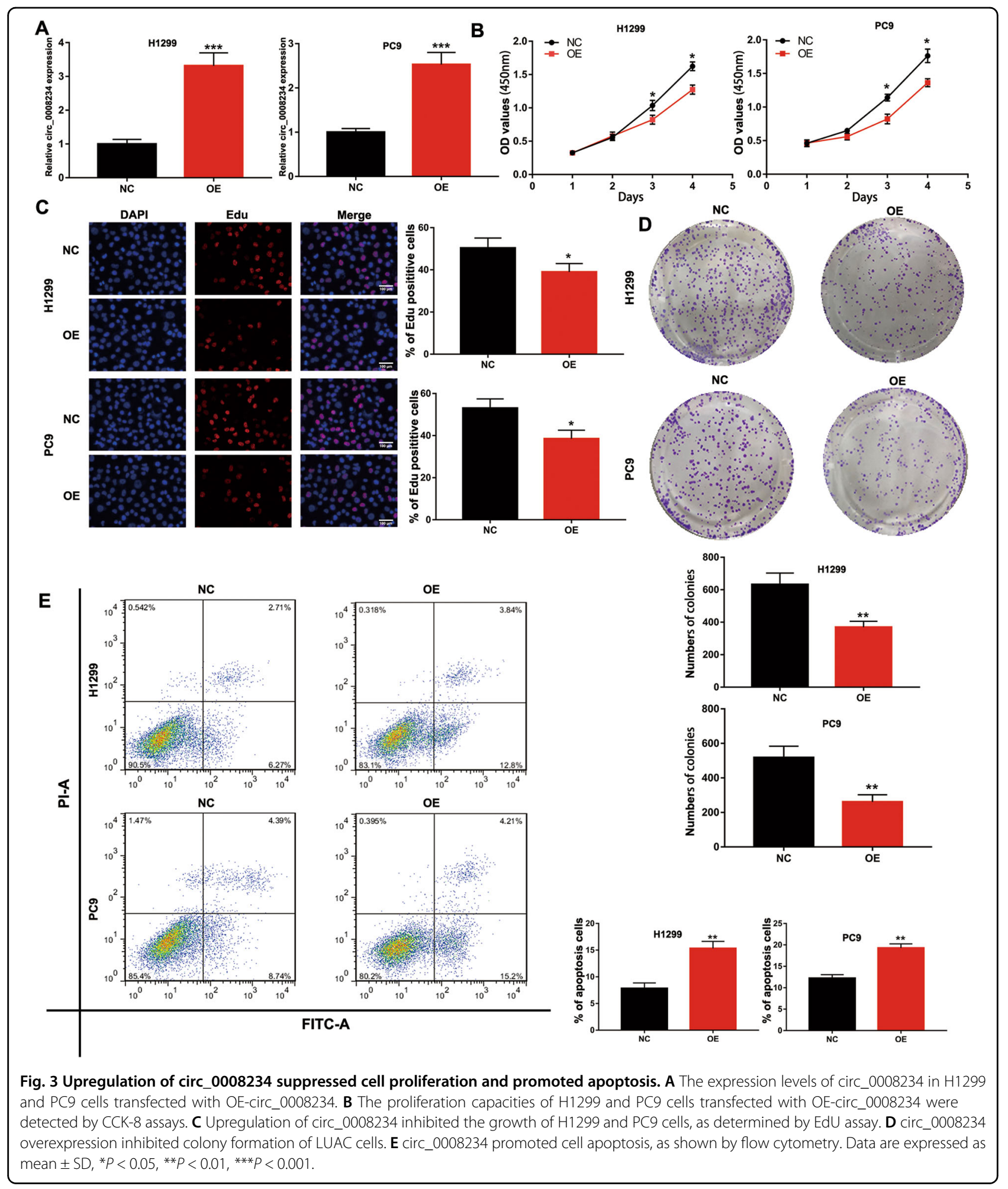

exogenously manipulate the expression of circ_0008234 in H1299 and PC9 cells. As shown in Fig. 3A, quantitative reverse transcriptase-PCR (qRT-PCR) results indicated that $\mathrm{OE}$ could specifically increase the expression of circ_0008234. On the one hand, the Cell Counting Kit-8 (CCK-8) assay reflected that the growth ability of LUAC cells was obviously decreased based on high circ_0008234 expression (Fig. 3B). On the other hand, as 5-ethynyl-2'- 
deoxyuridine (EdU) assays suggested, the proliferation rate of $\mathrm{H} 1299$ and PC9 cells was decreased by enhancing circ_0008234 expression (Fig. 3C). As shown in Fig. 3D, upregulation of circ_0008234 also reduced the colony formation ability of LUAC cells. Furthermore, circ_0008234 OE promoted apoptosis in H1299 and PC9 cells (Fig. 3E). Finally, an orthotopic xenograft mouse model was applied to determine the effect of circ_0008234 on tumorigenesis in vivo. The cells of the $\mathrm{NC}$ and $\mathrm{OE}$ groups were subcutaneously inoculated into the armpits of female nude mice. Nude mice were sacrificed 24 days later and photographed (Fig. S1A). After measuring the growth and volume of the tumor, we found that, compared with the $\mathrm{NC}$ group, both tumor growth and volume in the OE group were slower and smaller (Fig. S1B, C). The expression of circ_0008234 in the $\mathrm{OE}$ group was notably increased by using qRT-PCR (Fig. S1D). Further research also confirmed that, compared with the $\mathrm{NC}$ group, the positive rate of $\mathrm{Ki} 67$ in the $\mathrm{OE}$ group decreased, while the positive rate of cleavage caspase 3 increased (Fig. S1E). These experiments demonstrated that high expression of circ_0008234 inhibited proliferation and promoted apoptosis of LUAC cells.

\section{circ_0008234 could sponge miR-574-5p}

To further show the potential mechanism of circ_0008234, miR-574-5p was chosen from starBase 2.0 and Circular RNA Interactome (CRI; Fig. 4A). The predicted binding sites between circ_0008234 and miR-574$5 p$ are shown in Fig. 4B. To verify whether circ0008234 could directly bind to miR-574-5p, luciferase reporter gene plasmids were constructed including the circ0008234 wild-type sequence (wt-1) or mutant-type sequence (mut-1). The miR-574-5p mimics remarkably reduced the luciferase intensity of wt- 1 compared to mut1 (Fig. 4C). Furthermore, as indicated in Fig. 4D, E, miR574-5p expression was also notably higher in LUAC tissues and cell lines. When the expression of circ_0008234 increased, miR-574-5p expression was remarkably downregulated (Fig. 4F). Then RNA immunoprecipitation (RIP) analysis showed that, compared with immunoglobulin G immunoprecipitation, circ_0008234 and miR574-5p were remarkably enriched in Ago2 immunoprecipitation (Fig. 4G). In vitro functional assays showed that the growth rate of $\mathrm{H} 1299$ and PC9 cells after miR-574-5p mimic transfection was remarkably accelerated (Fig. $4 \mathrm{H}$ ).

\section{RND3 may be a potential target of miR-574-5p}

To determine the potential target of miR-574-5p, starBase 2.0, TargetScan, miRWalk, and miRDB were used, and 206 genes were found (Fig. 5A). After verification by The Cancer Genome Atlas (TCGA) and Clinical Proteomic Tumor Analysis Consortium (CPTAC) databases, RND3 was finally chosen (Fig. 5B). Another dual- luciferase reporter gene assay verified the possibility of complementary base pairing between miR-574-5p mimics and RND3 mRNA (Fig. 5C). At the same time, after OE of circ_0008234 in H1299 and PC9 cells, the mRNA and protein expression of RND3 was significantly upregulated (Fig. 5D). As shown in Fig. 5E, the mRNA and protein expression of RND3 in LUAC cells also decreased when miR-574-5p was overexpressed. These results suggested that circ_0008234 may regulate RND3 by sponging miR574-5p.

\section{Discussion}

Despite various advances and achievements in the diagnosis and treatment of LUAC, it remains one of the main causes of death globally ${ }^{17}$. The number of NSCLC cases has increased year by year, and the incidence of LUAC is the highest ${ }^{18}$. A growing number of studies have suggested that dysregulated circRNAs act as regulators in diverse diseases, including LUAC ${ }^{19-21}$.

In this manuscript, we identified a dysregulated circRNA (circ_0008234) in LUAC tissues through the GEO database. Low expression of circ_0008234 was first observed in LUAC tissues and cell lines. The expression level of circ_0008234 was closely correlated with tumor size, and circ_0008234 expression may be a predictor of prognosis. Functionally, circRNAs may play regulatory roles in LUAC. As recently reported, circRNA disorders can cause malignant behavior in human cancer cells ${ }^{22}$. Then in vitro and in vivo assays were designed and conducted to detect the proliferation and apoptosis rate of circ_0008234 in LUAC cell processes. After cell transfection, we determined that circ_0008234 OE would impede cell proliferation and accelerate apoptosis in LUAC.

Further research found that circ_0008234 could enhance the RND3 expression by modulating miR-5745p. miR-574-5p, as a member of the microRNA family, is closely tied to the occurrence and development of cancers. For example, miR-574-5p regulated cell invasion by regulating FOXN3 (forkhead box N3) in nasopharyngeal carcinoma and attenuated proliferation, migration, and the EMT pathway by modulating BCL11A (BAF chromatin remodeling complex subunit BCL11A) and SOX2 (SRY-box transcription factor 2) in triple-negative breast cancer $^{23,24}$. Furthermore, miR-574-5p was noticeably increased in the serum and tissue samples of early-stage NSCLC patients and promoted metastasis by modulating PTPRU (protein tyrosine phosphatase receptor type $\mathrm{U})^{25,26}$. In addition, the expression of miR-574-5p is regulated by long non-coding RNA (lncRNA) in cancers. For example, lncRNA PTCSC3 inhibited cervical carcinoma cell proliferation and invasion by sponging miR574-5p, and LINC00052 inhibited metastasis by regulating miRNA-574-5p in colorectal cancer ${ }^{27,28}$. However, the 


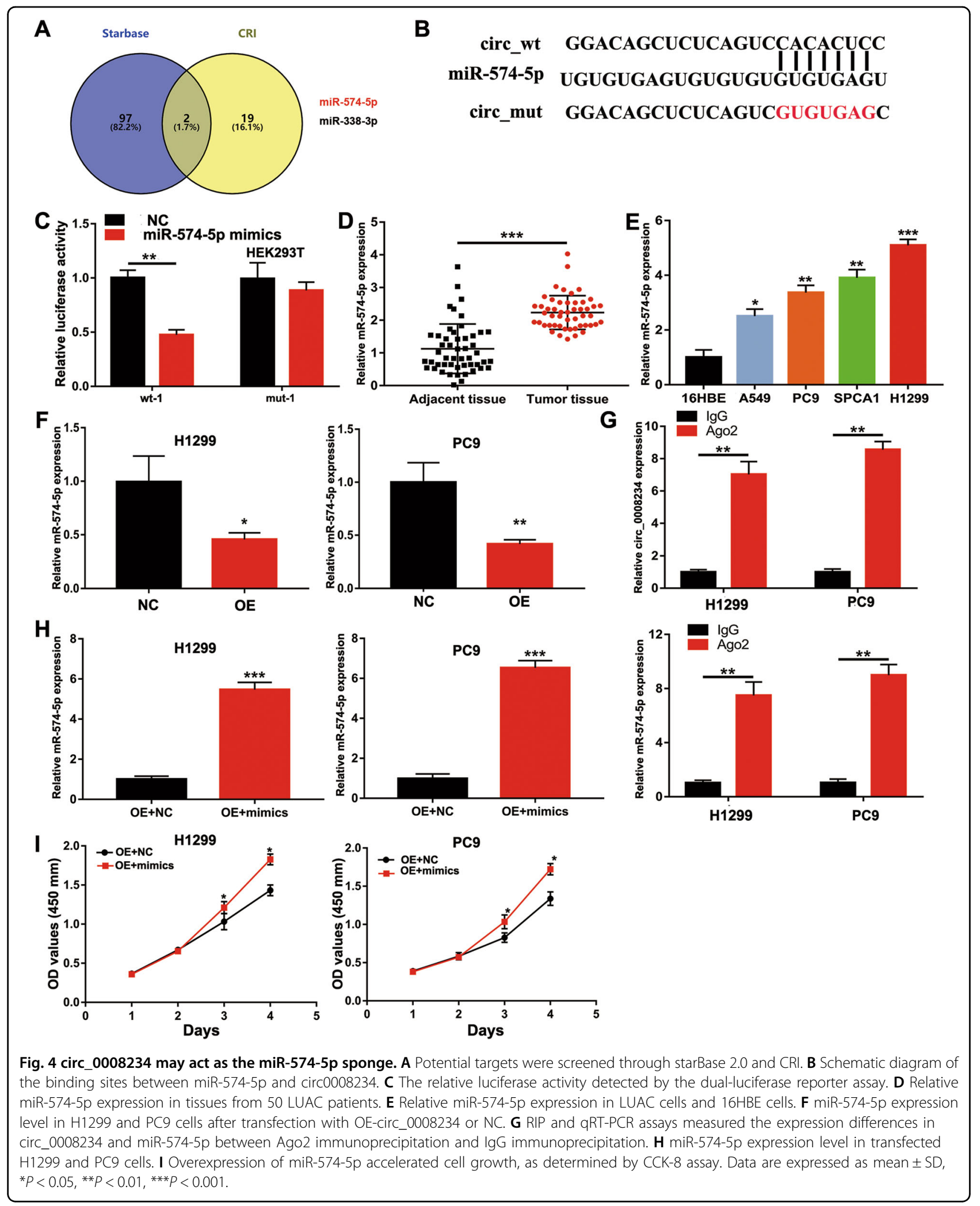




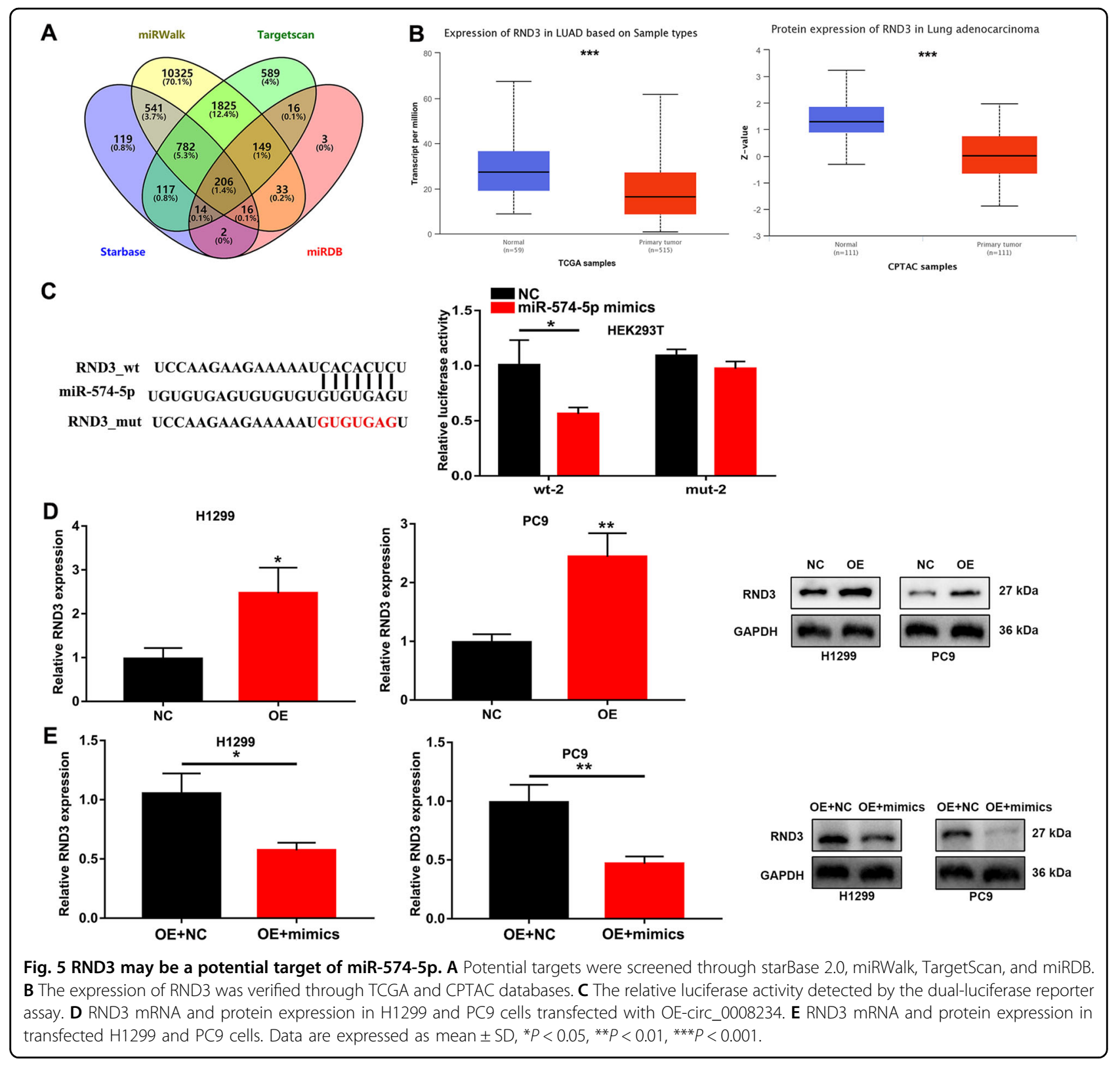

sponge adsorption effect of circRNA on miR-574-5p has not been studied. In this study, miR-574-5p was confirmed to be upregulated in LUAC tissues and cell lines, and circ_0008234 played a role as a tumor-suppressor gene by downregulating miR-574-5p. Through dualluciferase reporter gene assay and RIP assay, it was verified that circ_0008234 and miR-574-5p underwent direct complementary base pairing. Hence, we concluded that circ_0008234 led to a significant downregulation of miR574-5p.

Next, we also found a predicted target gene downstream of miR-574-5p: RND3. RND3 is a member of the RhoGTPase family and belongs to the RND subfamily, also known as $\mathrm{RhoE}^{29}$. RND3 participates in functions frequently regulated by Rho-GTPase, such as remodeling of the actin cytoskeleton, and numerous essential cellular processes, such as cell proliferation, differentiation, survival, motility, and adhesion ${ }^{30,31}$. These mechanisms may contribute to cancer development when dysregulated. Early research has shown that RND3 is overexpressed in NSCLC patients and is interconnected with poor prognosis, indicating that RND3 can be chosen as a prognostic marker for NSCLC patients ${ }^{32,33}$. However, recently generated functional data indicated that RND3 was downregulated in three NSCLC cell lines (one of which was a LUAC cell line), and the enhanced expression of RND3 inhibited cell proliferation ${ }^{34}$. The evaluation of RND3's impact on LUAC requires more in-depth research. In this 
manuscript, we verified the low expression of RND3 in LUAC tissues through the TCGA and CPTAC databases. Another dual-luciferase reporter assay indicated that there was a binding phenomenon between miR-574-5p and RND3 mRNA. The expression of RND3 mRNA was significantly upregulated when circ_0008234 was overexpressed. However, there was a negative relationship between the expression of RND3 and miR-574-5p. In summary, the above-mentioned results indicated that circ_0008234 could decelerate LUAC progression by serving as a miRNA sponge to regulate miR-574-5p RND3 expression.

Our manuscript highlights the potential of circ_0008234, miR-574-5p, and RND3 as novel therapeutic targets for treating LUAC patients and may help to further explore the specific mechanism of the ceRNA network in cancer. In future studies, we will combine these results with the application of traditional chemotherapy drugs or molecular-targeted antitumor drugs surgical patients or advanced patients to identify surgical opportunities to improve the prognosis and survival rate.

\section{Conclusion}

Our data indicate that circ_0008234 has a key part in the occurrence and development of LUAC, mainly through the "sponge effect" to modulate miR-574-5p expression, thereby promoting the mRNA expression of RND3. This research will provide new directions for improving and expanding molecular targets for the diagnosis and treatment of LUAC.

\section{Materials and methods}

\section{Bioinformatics analysis}

Microarray data were downloaded from the GEO database $^{35}$, including GSE112214 and GSE158695 ${ }^{36}$. Then the original microarray data were analyzed with GEO2R. All information on hsa_circ_0008234 comes from circBase (http://www.circbase.org/) ${ }^{37}$. The binding site information of miRNAs and circ_0008234 was derived from starBase 2.0 (http://www.starbase.sysu.edu.cn/starbase2/) and CRI (http://circinteractome.nia.nih.gov/) $)^{38,39}$. The binding site information of microRNA-574-5p and mRNAs was derived from starBase 2.0 (http://www.starbase.sysu.edu. cn/starbase2/), TargetScan (http://www.targetscan.org/), miRWalk (http://mirwalk.umm.uni-heidelberg.de/), and miRDB (http://mirdb.org/policy.html) ${ }^{38,40-42}$. TCGA and CTPAC were used to identify the expression of RND3 between tumor tissues and adjacent tissues ${ }^{43,44}$.

\section{LUAC tissues and cell lines}

Fifty tissue samples of LUAC patients have been obtained from the First Affiliated Hospital of Soochow University since June 2019. The clinical characteristics of the patients were recorded based on the American Joint
Committee on Cancer tumor/node/metastasis staging system (eighth edition). None of these patients received radiation or chemotherapy before surgical resection. LUAC cell lines (A549, SPCA1, H1299, and PC9), the 16HBE14o Human Bronchial Epithelial Cell Line (16HBE), and HEK293T cells were sourced from the Shanghai Academy of Sciences (Shanghai, China). All cells were moistened in RPMI 1640 medium (Gibco, NY, USA) supplemented with $10 \%$ fetal bovine serum (Gibco) and $100 \mathrm{U} / \mathrm{mL}$ penicillin and streptomycin (Gibco). Cells were cultured in an incubator under a $5 \% \mathrm{CO}_{2}$ atmosphere at $37^{\circ} \mathrm{C}$.

\section{Cell proliferation and apoptosis assay}

CCK-8, EdU, colony formation, and apoptosis assays were performed as described previously ${ }^{45}$.

\section{Statistical analysis}

Statistical analyses were performed by using the SPSS 25.0 software (IBM Corp., NY, USA). All means and standard deviations were calculated based on three independent experiments. Correlations between circ_008234 expression and the clinicopathological characteristics of the LUAC patients were examined using the $\chi^{2}$ test. Comparisons between two groups were analyzed using a two-sided Student's $t$ test. $P<0.05$ was considered statistically significant.

More details can be found in S2.

\section{Author contributions}

X.T., W.J., and Y.H. conceived the study and participated in the study design, performance, coordination, and manuscript writing. W.J., Y.H., Z.M., Y.Z., C.Z., and N.Z. performed the research. All authors have read and approved the final manuscript.

\section{Funding}

This study was sponsored by Natural Science Foundation of Xinjiang Uygur Autonomous Region (2021D01A187).

\section{Data availability}

Data supporting the present findings are available from the corresponding author upon reasonable request.

\section{Ethics statement}

The study was approved the ethics committee of the hospital, and informed consent was obtained from all patients.

Conflict of interest

The authors declare no competing interests.

\section{Publisher's note}

Springer Nature remains neutral with regard to jurisdictional claims in published maps and institutional affiliations.

Supplementary information The online version contains supplementary material available at https://doi.org/10.1038/s41420-021-00512-1.

Received: 1 February 2021 Revised: 24 April 2021 Accepted: 10 May 2021 Published online: 28 May 2021 


\section{References}

1. Yao, J., Xu, G., Zhu, L. \& Zheng, H. circGFRA1 enhances NSCLC progression by sponging miR-188-3p. Onco Targets Ther. 13, 549-558 (2020).

2. Siegel, R. L., Miller, K. D. \& Jemal, A. Cancer statistics, 2019. CA Cancer J. Clin. 69 7-34 (2019).

3. Zhang, B. et al. A novel technique for preoperative localization of pulmonary nodules using a mixture of tissue adhesive and iohexol under computed tomography guidance: a 140 patient single-center study. Thorac. Cancer 12, 854-863 (2021).

4. Gueguen, P. et al. Contribution of resident and circulating precursors to tumor-infiltrating CD8(+) T cell populations in lung cancer. Sci. Immunol. 6, eabd5778 (2021).

5. Jia, Q. et al. Mutational burden and chromosomal aneuploidy synergistically predict survival from radiotherapy in non-small cell lung cancer. Commun. Biol. 4, 131 (2021)

6. Wang, J. et al. circRNA-002178 act as a ceRNA to promote PDL1/PD1 expression in lung adenocarcinoma. Cell Death Dis. 11, 32 (2020).

7. Li, X., Yang, L. \& Chen, L. L. The biogenesis, functions, and challenges of circular RNAs. Mol. Cell 71, 428-442 (2018).

8. Kristensen, L. S., Hansen, T. B., Venø, M. T. \& Kjems, J. Circular RNAs in cancer: opportunities and challenges in the field. Oncogene 37, 555-565 (2018).

9. Ying, X., Zhu, J. \& Zhang, Y. Circular RNA circ-TSPAN4 promotes lung adenocarcinoma metastasis by upregulating ZEB1 via sponging miR-665. Mol. Genet. Genom. Med. 7, e991 (2019).

10. Salmena, L., Poliseno, L., Tay, Y., Kats, L. \& Pandolfi, P. P. A ceRNA hypothesis: the Rosetta Stone of a hidden RNA language? Cell 146, 353-358 (2011).

11. Zhong, Y. et al. Circular RNAs function as ceRNAs to regulate and control human cancer progression. Mol. Cancer 17, 79 (2018).

12. Huang, G. et al. CircRNA hsa_circRNA_104348 promotes hepatocellular carcinoma progression through modulating miR-187-3p/RTKN2 axis and activating Wnt/B-catenin pathway. Cell Death Dis. 11, 1065 (2020).

13. Wang, X. \& Zhu, Y. Circ_0000020 elevates the expression of PIK3CA and facilitates the malignant phenotypes of glioma cells via targeting miR-142-5p. Cancer Cell Int. 21, 79 (2021).

14. Yu, Z. et al. Circ-HMGA2 (hsa_circ_0027446) promotes the metastasis and epithelial-mesenchymal transition of lung adenocarcinoma cells through the miR-1236-3p/ZEB1 axis. Cell Death Dis. 12, 313 (2021).

15. Yao, Y., Zhou, Y. \& Hua, Q. circRNA hsa_circ_0018414 inhibits the progression of LUAD by sponging miR-6807-3p and upregulating DKK1. Mol. Ther. Nucleic Acids 23, 783-796 (2021).

16. Wang, S. et al. Circular RNA FOXP1 promotes tumor progression and Warburg effect in gallbladder cancer by regulating PKLR expression. Mol. Cancer 18, 145-145 (2019).

17. Aggarwal, A. et al. The state of lung cancer research: a global analysis. J. Thorac. Oncol. 11, 1040-1050 (2016).

18. $\mathrm{Xu}, \mathrm{X}$. et al. c-Met and CREB1 are involved in miR-433-mediated inhibition of the epithelial-mesenchymal transition in bladder cancer by regulating Akt/ GSK-3beta/Snail signaling. Cell Death Dis. 7, e2088 (2016).

19. Wang, X. et al. Decreased expression of hsa_circ_001988 in colorectal cancer and its clinical significances. Int. J. Clin. Exp. Pathol. 8, 16020-16025 (2015).

20. Zhong, Z., LV, M. \& Chen, J. Screening differential circular RNA expression profiles reveals the regulatory role of circTCF25-miR-103a-3p/miR-107-CDK6 pathway in bladder carcinoma. Sci. Rep. 6, 30919 (2016).

21. $\mathrm{Xu}, \mathrm{L}$. et al. The circular RNA ciRS-7 (Cdr1as) acts as a risk factor of hepatic microvascular invasion in hepatocellular carcinoma. J. Cancer Res. Clin. Oncol. 143, 17-27 (2017)

22. Qu, S. et al. Circular RNA: a new star of noncoding RNAs. Cancer Lett. 365, 141-148 (2015).
23. Lin, Z., Chen, M., Wan, Y., Lei, L. \& Ruan, H. miR-574-5p targets FOXN3 to regulate the invasion of nasopharyngeal carcinoma cells via Wnt/ $\beta$-catenin pathway. Technol. Cancer Res. Treat. 19, 1533033820971659 (2020).

24. Zhang, K. J. et al. miR-574-5p attenuates proliferation, migration and EMT in triple-negative breast cancer cells by targeting BCL11A and SOX2 to inhibit the SKIL/TAZ/CTGF axis. Int. J. Oncol. 56, 1240-1251 (2020).

25. Zhou, R. et al. MicroRNA-574-5p promotes metastasis of non-small cell lung cancer by targeting PTPRU. Sci. Rep. 6, 35714 (2016).

26. Foss, K. M. et al. miR-1254 and miR-574-5p: serum-based microRNA biomarkers for early-stage non-small cell lung cancer. J. Thorac. Oncol. 6, 482-488 (2011).

27. Zhang, M., Song, Y. \& Yu, L. LncRNA PTCSC3 suppressed cervical carcinoma cell invasion and proliferation via regulating miR-574-5p. Am. J. Transl. Res. 11, 7186-7194 (2019)

28. Yu, G. et al. Long noncoding RNA LINC00052 inhibits colorectal cancer metastasis by sponging microRNA-574-5p to modulate CALCOCO1 expression. J. Cell. Biochem. 120, 17258-17272 (2019).

29. Tang, Q., Li, M., Chen, L., Bi, F. \& Xia, H. miR-200b/c targets the expression of RhoE and inhibits the proliferation and invasion of non-small cell lung cancer cells. Int. J. Oncol. 53, 1732-1742 (2018).

30. Cario-Toumaniantz, C. et al. Modulation of RhoA-Rho kinase-mediated Ca2+ sensitization of rabbit myometrium during pregnancy - role of Rnd3. J. Physiol. 552, 403-413 (2003).

31. Georgess, D. et al. Comparative transcriptomics reveals RhoE as a novel regulator of actin dynamics in bone-resorbing osteoclasts. Mol. Biol. Cell 25, 380-396 (2014).

32. Zhang, C. et al. Overexpression of RhoE has a prognostic value in non-small cell lung cancer. Ann. Surg. Oncol. 14, 2628-2635 (2007).

33. Cuiyan, Z. et al. Overexpression of RhoE in non-small cell lung cancer (NSCLC) is associated with smoking and correlates with DNA copy number changes. Cancer Biol. Ther. 6, 335-342 (2007).

34. Tang, $Y$. et al. Rnd3 regulates lung cancer cell proliferation through notch signaling. PLOS ONE 9, e111897 (2014).

35. Clough, E. \& Barrett, T. The Gene Expression Omnibus Database. Methods Mol Biol. 1418, 93-110 (2016).

36. Li, B. et al. circNDUFB2 inhibits non-small cell lung cancer progression via destabilizing IGF2BPs and activating anti-tumor immunity. Nat. Commun. 12, 295 (2021).

37. Glazar, P., Papavasileiou, P. \& Rajewsky, N. circBase: a database for circular RNAs. RNA 20, 1666-1670 (2014).

38. Li, J. H., Liu, S., Zhou, H., Qu, L. H. \& Yang, J. H. starBase v2.0: decoding miRNAceRNA, miRNA-ncRNA and protein-RNA interaction networks from large-scale CLIP-Seq data. Nucleic Acids Res. 42, D92-D97 (2014).

39. Dudekula, D. B. et al. Circlnteractome: a web tool for exploring circular RNAs and their interacting proteins and microRNAs. RNA Biol. 13, 34-42 (2016).

40. Mon-Lopez, D. \& Tejero-Gonzalez, C. M. Validity and reliability of the TargetScan ISSF Pistol \& Rifle application for measuring shooting performance. Scand. J. Med. Sci. Sports 29, 1707-1712 (2019).

41. Sticht, C., De La Torre, C., Parveen, A. \& Gretz, N. miRWalk: an online resource for prediction of microRNA binding sites. PLOS ONE 13, e0206239 (2018).

42. Chen, Y. \& Wang, X. miRDB: an online database for prediction of functional microRNA targets. Nucleic Acids Res. 48, D127-D131 (2020).

43. Chang, J. T., Lee, Y. M. \& Huang, R. S. The impact of the Cancer Genome Atlas on lung cancer. Transl. Res. 166, 568-585 (2015).

44. Rudnick, P. A. et al. A description of the Clinical Proteomic Tumor Analysis Consortium (CPTAC) common data analysis pipeline. J. Proteome Res. 15 1023-1032 (2016).

45. Jiang, W. et al. MicroRNA-1258 suppresses tumour progression via GRB2/Ras/ Erk pathway in non-small-cell lung cancer. Cell Prolif. 51, e12502 (2018). 